\title{
Aplicação da metodologia DFMA para elaboração de um Projeto de Melhoria em Processo Produtivo
}

\section{Application of the DFMA methodology for the development of a production process improvement project}

\author{
Alexandre Formigoni ${ }^{1,2 *}$, Vanderlei Tallach ${ }^{1,2}$, João Roberto Maiellaro ${ }^{1}$; Rosinei Batista \\ Ribeiro $^{2}$; Eliane Antonio Simões²; Helena Gemignani Peterossi²; Fabricio José Piacente ${ }^{2}$
}

\begin{abstract}
RESUMO
A busca por melhoria contínua dos índices de produtividade demanda que as organizações estejam em constante análise e atualização dos seus processos produtivos. Com o advento da manufatura enxuta, muitas ferramentas foram desenvolvidas e colocadas à disposição dos pesquisadores e profissionais. Compreender o processo produtivo aliado ao domínio das ferramentas, proporciona melhores condições para elaborar um projeto de manufatura enxuta integrado ao projeto do produto. Para início da pesquisa, foi analisado o relatório mensal de produção, extraído automaticamente do sistema ERP da empresa estudada. Foi possível identificar e selecionar o produto com maior atraso de entrega, e iniciou-se a análise de todo o processo produtivo e movimentações de componentes por meio do uso do método Design for Manufacturing and Assembly (DFMA), com o uso das ferramentas Value Stream Mapping (VSM), Estudo de tempos e movimentos e Análise do arranjo físico. A interpretação dos resultados obtidos possibilitou a elaboração de um projeto de manufatura enxuta compatível com os níveis de demanda e capacidade instalada, assegurando o cumprimento das demandas e redução gradativa dos atrasos, até sua completa eliminação após 2 meses. Incorporado aos ganhos, constatou-se aumento de $28 \%$ do índice de valor agregado ao processo produtivo. Os demais resultados obtidos com as ações propostas e implementadas, estão detalhados no corpo deste artigo, evidenciando que a implantação de uma metodologia adequada, suportada por ferramentas específicas podem alavancar a produtividade, tornando o processo enxuto e rentável.
\end{abstract}

Palavras-chave: DFMA; Produção enxuta; Melhoria contínua.

\footnotetext{
ABSTRACT

The search for continuous improvement in productivity indexes demands that organizations are constantly analyzing and updating their production processes. With the advent of lean manufacturing, many tools were developed and made available to researchers and professionals. Understanding the process combined with mastering the tools provides better conditions to develop a lean manufacturing project integrated to the product design. To start the research, the monthly production report, automatically extracted from the ERP system of the studied company, was analyzed. It was possible to identify and select the product with the

${ }^{1}$ Faculdade de Tecnologia do Estado de São Paulo, Unidade de Guarulhos, Centro Estadual de Educação Tecnológica Paula Souza - CEETEPS - São Paulo, Brasil.

*E-mail: alexandre.formigoni@cpspos.sp.gov.br

${ }^{2}$ Programa de Mestrado Profissional Gestão e Tecnologia em Sistemas Produtivos - Unidade de Pós-Graduação, Extensão e Pesquisa - Centro Estadual de Educação Tecnológica Paula Souza CEETEPS - São Paulo, Brasil.
} 
longest delivery delay and began the analysis of the entire production process and component movements through the use of Design for Manufacturing and Assembly (DFMA), Value Stream Mapping (VSM) tools. Study of times and methods and Analysis of the physical arrangement. The interpretation of the obtained results allowed the elaboration of a lean manufacturing project compatible with the levels of demand and installed capacity, ensuring the fulfillment of demands and a gradual reduction of delays, until its complete elimination after 2 months. Incorporated into the gains, there was an increase of $28 \%$ in the value-added index to the production process. The other results obtained with the proposed and implemented actions are detailed in the body of this article, showing that the implementation of an adequate methodology, supported by specific tools, can leverage productivity, making the process lean and profitable.

Keywords: Design for manufacturing and assembly; Lean production; Continuous improvement.

\section{INTRODUÇÃO}

Para se obter vantagem e pioneirismo em um mercado em mudança, as empresas de manufatura precisam de melhores métodos e ferramentas que atendam a customização em massa durante as fases iniciais de desenvolvimento (FERGUSON et al. 2013).

A necessidade de atender rapidamente as demandas do cliente, exige que as empresas de manufatura mantenham alta produtividade e consigam mitigar os distúrbios da produção (BOKRANTZ et al. 2016).

Além de atender as necessidades dos clientes, os produtos precisam ser adaptados levando em consideração as capacidades dinâmicas da manufatura durante as etapas iniciais do desenvolvimento.

Koufteros et al. (2014) estudaram a correlação entre as plataformas de produto, engenharia simultânea e métodos de fabricação e observaram que o desempenho da empresa é afetado pelas estratégias de desenvolvimento de produto e pelas práticas de manufatura.

Por meio de observação no processo produtivo em uma empresa de manutenção em máquinas portáteis de pagamento, foi diagnosticado que a capacidade produtiva da operação era insuficiente em relação à demanda, que havia excesso de materiais e componentes ao longo do processo e que não havia um fluxo contínuo de operação. Diante deste cenário, levantou-se a dúvida sobre o método utilizado para o dimensionamento do quadro de funcionários, se as etapas do processo foram definidas seguindo algum conceito de produção enxuta, se o leiaute do processo possibilita reduzir movimentação de materiais e pessoas, e, se seria possível aumentar a produtividade reduzindo a mão de obra simultaneamente. 
O presente trabalho tem o objetivo de analisar por meio da aplicação de um conjunto de métodos e ferramentas de gestão, os cenários de implantação e a otimização do processo de manufatura.

Para elaboração do estudo, foram coletadas informações detalhadas da manufatura, analisados os dados e elaborado um projeto de processo.

Ao final os resultados demonstram que com a aplicação dos métodos e ferramentas, foi possível propor um novo processo produtivo, com capacidade de atender a demanda do cliente a um menor custo.

\section{REFERENCIAL TEÓRICO}

\section{Design for Assembly (DFA)}

DFA é um método para aperfeiçoamento de projetos para uma montagem simples e de custo mínimo (KIM, BEKEY E GOLDBERG, 1992). O objetivo deste método é tornar o processo mais simples e reduzir o custo total além do tempo gasto para montagem (TODIC, VUKMAN, 2012).

A simplicidade do processo de montagem não depende unicamente do item e suas características, mas depende do processo de abastecimento de materiais, manuseio e procedimento de montagem (BOOTHROYD,1994).

O DFA proporciona aos engenheiros de projeto, conhecimento sobre questões de montagem, permitindo antecipação e melhoria junto ao projeto (KRUMENAUER, MATAYOSHI, FILHO e BATALHA, 2008).

O DFA também é uma espécie de visão de mundo do desenho, com o qual o especialista utiliza uma vasta gama de estratégias para analisar, estimar, organizar e reproduzir cada um dos elementos que vai influenciar o procedimento de montagem durante toda a fase de desenho, a fim de que se reduza significativamente os custos envolvidos na montagem (DRISCOLL, 2002).

Em um estudo de DFA, critérios como reduzir e aperfeiçoar a contagem e classificação de peças, eliminar a necessidade de modificações, projetar peças que sejam de fácil localização e ajustes, garantir simplicidade e segurança no manuseio e montagem dos componentes, minimizar a quantidade de instrumentos para montagem, devem ser considerados.

As vantagens diretas representam diminuição dos custos fixos e variáveis de 
montagem, sendo elas manuais ou não. Um item projetado utilizando os princípios de DFA demandam necessidade de menos máquinas, especialistas para montagem e reduz as dificuldades das atividades de montagem.

Vantagens indiretas são a simplificação do controle de estoque, aumento da confiabilidade do item reduzindo o tempo de garantia e custos de manutenção (KRUMENAUER, MATAYOSHI, FILHO e BATALHA, 2008).

\section{Design for Manufacturing (DFM)}

O método DFM trata do conhecimento sobre como os dados do processo se conectam com modelos enxutos olhando para as restrições ligadas a manufatura, no período inicial do projeto. Vantagens essenciais da utilização do DFM são melhor qualidade do projeto, redução de despesas e planejamento otimizado de mudanças. Aprimorar o tempo de criação juntamente com projeto, inovação, montagem e utilidade e reduzir despesas de custo com alta tecnologia, manutenção e montagem, são considerados como objetivos principais do método.

A utilização de práticas do DFM, ilustrou a forma ordenada e efetiva de execução do método em situações de fabricação. (DRISCOLL, 2002). Projetistas têm de considerar a capacidade de fabricação para atender os objetivos de simplificar o processo de fabricação e alinhar o projeto com o procedimento para obter resultados ideais (BOOTHROYD,1994).

Para execução do DFM regras como reduzir quantidade e variedade de componentes, utilizar componentes intercambiáveis, projetar para facilitar a fabricação, e projetar componentes que tenham diferentes funções, são fundamentais para o sucesso.

\section{Design for Manufacturing and Assembly (DFMA)}

A palavra DFMA origina da combinação de DFM e DFA, que combinam dois fatores fundamentais no desenho do item mantendo a utilidade e aprimorando o procedimento de design de produto.

Os estudos ajudam a lidar com os custos de fabricação, capacitando projetistas a ativos de forma mais efetiva, reduzindo custo e aprimorando os indicadores do negócio.

Uma visão geral do procedimento para análise do DFMA é identificar o produto no qual a análise será realizada. Após a seleção, o produto é individualmente desmontado, as peças são modeladas em software CAD.

A análise de custo de DFM é realizada nas peças individuais para definir uma 
linha de base de custo de desenho do produto e a análise de DFA para identificar os candidatos a eliminação (TODIC, VUKMAN, 2012).

De acordo com Selvaraj, Radhakrishnan e Adithan (2008) a maioria dos problemas na manufatura e montagem ocorrem devido à abordagem centrada no projeto do produto que ignora os problemas do sistema de fabricação e montagem. Os fatores que contribuem para o erro durante o projeto incluem variações inadequadas nas peças, uso incorreto de dados de fabricação, máquinas, métodos de fixação e ferramentas usadas na fabricação de componentes, projeto incorreto de acessórios, localização incorreta além do fato de não se considerar o desgaste das ferramentas e efeitos ambientais e a falta de habilidades, treinamento e experiência dos operadores, questões críticas durante a fabricação e montagem.

Facilidade de manuseio, peças estáveis e autocompensadoras, tolerância flexível, menos fixadores, ferramentas e acessórios, apresentação das peças na orientação correta e contagem de peças são algumas das considerações do DFMA que reduzirão o tempo e custo de desenvolvimento do produto. O fator de custo para uma montagem é expressivo e qualquer redução nessa atividade terá uma redução substancial no custo do produto (SELVARAJ, RADHAKRISHNAN e ADITHAN, 2008).

Selvaraj, Radhakrishnan e Adithan (2008) apresentam ainda a importância da redução do retrabalho e das horas extras associadas aos ajustes, calços e recortes durante a montagem e afirmam que o design pode ajudar a atingir o mínimo de ferramentas na fabricação e montagem por meio da abordagem sistemática da integração de design de produtos e seus processos relacionados, incluindo fabricação e fornecimento. Eles explicam DFM e DFA como um dos métodos de obtenção de engenharia simultânea, permitindo que o design de um produto seja fácil de fabricar, montar e ter uma boa relação custo-benefício.

\section{Mapeamento de Fluxo de Valor - VSM}

Mapeamento do fluxo de valor, Value Stream Mapping (VSM) é uma ferramenta eficaz por suas características em mapear todo o fluxo do processo e seu valor agregado, e identificar as etapas que não agregam valor na fabricação de um produto ou serviço, do fornecedor ao cliente (JIN, DOOLEN, 2014).

O VSM mostra como cada processo é, com o momento de proceder as necessidades do cliente do próximo processo, com todos os movimentos de materiais e informação. As fontes de perdas são identificadas e no planejamento do estado futuro são 
eliminadas, considerando toda a cadeia de valor e não apenas os processos individuais locais (LIBRELATO et al. 2014).

Em qualquer indústria de transformação, as operações podem ser classificadas em três gêneros principais: atividades que não agregam valor (ANVN), mas necessárias; atividades que não agregam valor e desnecessárias (ANVD) e as que agregam valor (AV).

As atividades (ANVD) não agregam valor ao cliente e nem à empresa, se constituem como desperdício e precisam ser erradicadas (manuseio desnecessário, reprocesso, tempo de espera etc.). Para apresentação dos processos de produção de forma esquemática, são utilizados símbolos que representam produtos, operações e fluxos de informação (JASTI, SHARMA, 2014).

\section{Arranjo Físico}

Diversas razões podem motivar a melhoria do arranjo físico em plantas industriais como, necessidade de expansão da capacidade produtiva, introdução de nova linha de produtos, segurança, economia de movimentos ou melhoria do ambiente de trabalho.

Alguns viabilizam a flexibilidade tornando possível a mudança sempre que necessário, outros possibilitam a verticalização otimizando a ocupação do espaço, comunicação visual sinalizando o fluxo de circulação, ergonomia proporcionando conforto para o funcionário e acessibilidade para atividades de manutenção e limpeza.

Os arranjos são classificados por produto quando é projetado por linha de montagem, por tipo de processo ou funções similares, por células quando se agrupam atividades similares e leiaute fixo. (SLACK, CHAMBERS e JOHNSTON, 2009).

As decisões sobre o tipo de arranjo a ser desenvolvido são tomadas em níveis estratégicos, sobretudo se resultar em grandes investimentos. Outrossim, se o leiaute não for bem projetado, pode gerar impactos negativos para a empresa e para o processo produtivo requerendo ações cautelosas pela alta direção, (MAYNARD, 1970; MARTINS, LAUGENI,1998; CORRÊA, CORRÊA, 2012 E SLACK, CHAMBERS E JOHNSTON, 2009)

\section{Tempos e Movimentos}

A humanidade, desde as suas origens nos tempos mais remotos, vem sendo acompanhada pela função produção, que pode ser entendida como um conjunto de atividades que levam a transformação de um bem tangível em outro com maior utilidade (MARTINS, LAUGENI, 2015) 
Segundo Slack, e Johnston (2013), a agilidade na tomada de decisões, na movimentação de materiais e comunicação interna da operação é muito importante proporcionando benefícios como redução de estoques, redução de riscos e rapidez na resposta aos clientes externos. Em contrapartida, o desperdício ou o uso inadequado do tempo pode se transformar em custos extras durante a operação.

No início a força humana foi substituída pelas máquinas a vapor e os artesãos começaram a realizar seus trabalhos nas primeiras fábricas. Houve uma revolução nos produtos e seus processos de fabricação, criaram-se os primeiros indicadores, técnicas de PCP, planejamento e controle financeiro e desenvolvimento de técnicas de vendas (MARTINS; LAUGENI, 2015).

Segundo Peinado e Graeml (2007), o cálculo do trabalho de forma científica, com uso de técnicas estatísticas, começou na primeira metade do século XX e era aplicada apenas nas indústrias. Os pioneiros na área foram Frederick W. Taylor, o casal Frank e Lílian Gilbreth, Henry Ford e Henry L. Gantt.

O objetivo da medição dos tempos de trabalho era definir qual a melhor maneira de desenvolvimento de uma tarefa específica, metodologia que permanece praticamente inalterada desde aquela época. A cronometragem das tarefas continua sendo largamente utilizada na maioria das empresas brasileiras com o objetivo de medir e avaliar o desempenho do trabalho (PEINADO, GRAEML, 2007).

Segundo Gaither e Frazier (2004), pode-se dizer que a medida do trabalho está relacionada ao processo de estimar a quantidade de tempo que um operador necessita para gerar uma unidade de produção e sua meta final quase sempre é desenvolver padrões de mão-de-obra com alta produtividade.

Slack, e Johnston (2013), afirmam que o estudo de tempos consiste em uma técnica de medição do trabalho muito utilizada para fins registro dos tempos e andamento do trabalho para elementos de determinada tarefa, geralmente realizada em condições prédeterminadas, os dados obtidos são analisados para se obter o tempo que é necessário para a realização do trabalho e definir seu nível de desempenho.

De acordo com Peinado e Graeml (2007), a amostragem do trabalho é uma técnica que permite estimar a porcentagem de tempo que um trabalhador ou uma máquina utiliza em cada atividade, não é necessário a observação contínua nem cronometrar a atividade. Em 1940, nos EUA, foi implantada com o nome de relação de esperas, seu uso ganhou mais popularidade a partir de 1950. Atualmente, é mais utilizada que a cronometragem, 
posto que o aumento do número de organizações de serviços precisa de técnicas com rapidez e maior abrangência (PEINADO, GRAEML,2007)

\section{Meios de Pagamento}

O uso de meios eletrônicos de pagamento vem se modificando no Brasil e no mundo, com tendência de crescimento desde o início do século XXI e tem importância ainda maior nas compras remotas, auxiliando no desenvolvimento desse setor econômico (LEÃO, SOTTO, 2019).

As adquirentes, termo que faz parte do jargão do setor bancário, popularmente chamadas de "máquinas de cartão", que contribuem com a eficiência dos serviços bancários, que foram os primeiros a adotar esse meio de pagamento em 1995. Em 2010, o Bando Central do Brasil fomentou a desverticalização dessa atividade ampliando a oferta de sistemas e equipamentos (MATTOS, 2017).

Estima-se que, no Brasil, 76,1\% da população acima de 18 anos possua cartão com meio de pagamentos, apesar de que as taxas para manter cartões são consideradas altas. Para os empreendedores, a aceitação do cartão não é mais vantagem competitiva, sendo muitas vezes questão de sobrevivência (CARMO, 2017).

\section{MÉTODO}

Este trabalho foi desenvolvido em uma empresa de remanufatura de máquinas para meios de pagamento, especificamente na linha de produção do equipamento (máquina de pagamento) que, por questões de confidencialidade, será denominado por modelo "A". A análise foi realizada neste modelo, devido ao problema crucial da empresa, que não conseguia produzir, a quantidade de peças demandada pelo cliente, mensalmente.

Inicialmente foram realizadas observações e entrevistas informais com os administradores e operadores nos locais de fabricação do modelo A e numa análise preliminar pode-se constatar:

a) o processo de montagem era muito demorado e complexo;

b) existiam muitas operações redundantes e excesso de movimentação de materiais entre os operadores;

c) a linha de produção foi implantada sem considerar os critérios para produção contínua seriada; 
d) não existia um leiaute adequado para montagem dos componentes;

e) a sequência das atividades havia sido definida através de informações passadas pela experiência dos operadores;

f) a quantidade de operadores não era dimensionada com base na análise de tempo e estudo de movimentos.

Diante deste cenário, constatou-se a necessidade de otimizar o processo produtivo do modelo A, para identificar e eliminar os gargalos, bem como, reduzir o tempo de ciclo e de atravessamento, criando um modelo de processo produtivo mais simples e otimizado.

A seleção da metodologia DFMA se deu devido a necessidade de simplificar o processo produtivo, reduzir o custo de produção, reduzir a quantidade de componentes e otimizar a sequência das atividades. Como nessa metodologia, o conceito da abordagem simultânea exige o uso de ferramentas que permitam a análise conjunta do produto, processo de fabricação e montagem, definiu-se pela utilização de 3 ferramentas:

a) VSM (Value Stream Mapping);

b) Estudo de Tempos e Movimentos; e

c) Estudos de Arranjos Físicos (Leiautes).

Na Figura 1 apresenta-se o delineamento da pesquisa, iniciando com a questão de pesquisa e a partir dessa, os passos dados para sua solução.

Figura 1 - Delineamento da Pesquisa com o uso das questões e problemas na investigação com o DFMA

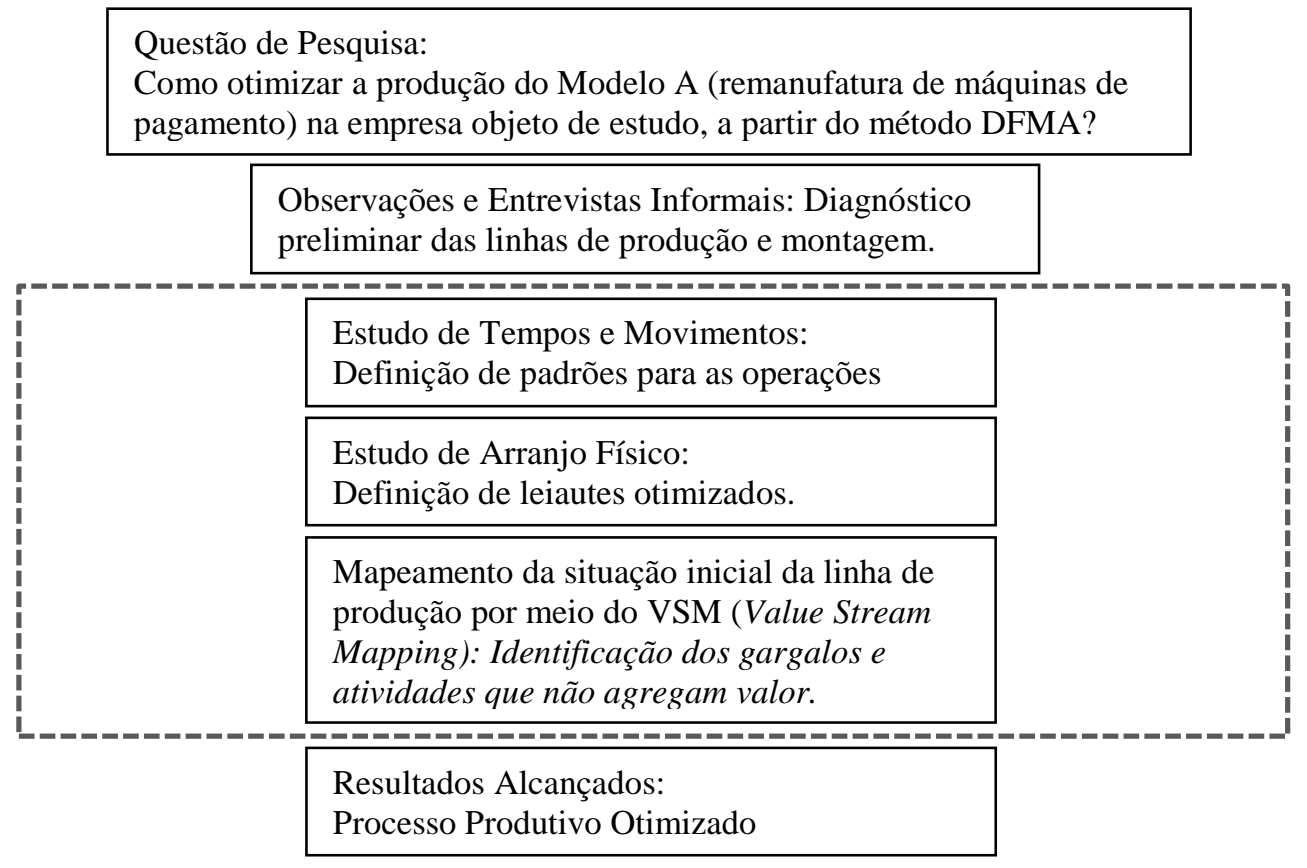

Fonte: Autores (2021) 
Iniciou-se com o estudo de tempos e movimentos por meio da observação física na linha de montagem, com tomada de 10 tempos para cada atividade para obtenção do tempo padrão. Em seguida foi elaborado o projeto de cada posto de trabalho, considerando as particularidades de cada atividade e operador, proporcionando que o operador pudesse executar a atividade com menor esforço físico possível e dentro novo tempo padrão calculado, reduzindo assim, o tempo de atravessamento.

O mapeamento do processo foi feito com utilização da ferramenta da qualidade VSM (Value Stream Mapping), aplicada em todas as linhas de produção, o que permitiu entender de maneira eficiente o tempo de cada atividade, possibilitando implementar melhorias para otimizar as etapas do processo, reduzindo o tempo de produção.

\section{RESULTADOS E DISCUSSÃO}

A linha de produção existente havia sido projetada e construída para a fabricação de cada equipamento individualmente, isto devido a particularidade de sequenciamento de etapas e diversidade de processos.

Ao estudar as etapas de produção, foi identificado que algumas delas estavam com o tempo de processo desbalanceado entre os operadores, ou seja, não havia um tempo padrão a ser adotado como parâmetro entre os operadores, cada operador tinha a sua meta diária estabelecida como padrão.

Além disto, foi verificado que cada operador individualmente, tinha seu método de produzir para obter maior volume de produção diário, o que ocasionava gargalos de posição entre os operadores e, no final do turno, o volume total necessário a ser produzido não era atingido e horas extras eram programadas para o atingimento da meta diária de produção.

$\mathrm{Na}$ tentativa de solucionar esta situação, foi implantado um turno adicional iniciando a jornada às $14 \mathrm{hs}$. Essa proposta de solução a princípio apresentou-se como viável. Entretanto, ao analisar os custos incorridos e calcular a produtividade, foi constatado que não seria economicamente a melhor alternativa. Optou-se então pela implementação simultânea das ferramentas de VSM, estudo de tempos e movimentos e estudo de leiaute. Todas elas com o objetivo de alavancar o volume produzido e reduzir os custos simultaneamente.

A Figura 2 apresenta os resultados comparativos entre o total de operadores e a 
quantidade de operações obtidos com a implementação das ferramentas, tendo sido obtida uma redução de $33 \%$ dos operadores sendo que, para alguns equipamentos o total de operadores se manteve e no equipamento " $G$ " houve a necessidade de se aumentar o quadro. Analogamente, observa-se a redução global de 21 operações significando redução de $14 \%$ do total, evidenciando que poder-se-ia eliminar o turno adicional e as horas extraordinárias. O resultado do estudo do leiaute, proporcionou uma redução significativa das movimentações dos equipamentos, otimizando o tempo de atravessamento (TA) e reduzindo o tempo de ciclo (TC), permitindo superar a demanda do cliente resultando em aumento da demanda em meses posteriores.

Figura 2 - Comparativo da quantidade de operadores e total de operações antes e depois da implantação das melhorias

\begin{tabular}{|c|c|c|c|c|}
\cline { 2 - 5 } \multicolumn{1}{c|}{} & \multicolumn{2}{c|}{ OPERADORES } & \multicolumn{2}{c|}{$\mathbf{N}^{\mathbf{0}}$ OPERAÇ OES } \\
\hline EQUIPAMENTO & INICIAL & FINAL & INICIAL & FINAL \\
\hline A & 53 & 34 & 12 & 10 \\
\hline B & 24 & 18 & 12 & 10 \\
\hline C & 25 & 15 & 12 & 9 \\
\hline D & 8 & 6 & 12 & 10 \\
\hline E & 4 & 3 & 10 & 10 \\
\hline F & 12 & 11 & 12 & 7 \\
\hline G & 15 & 18 & 11 & 12 \\
\hline H & 5 & 5 & 7 & 7 \\
\hline I & 2 & 2 & 6 & 5 \\
\hline J & 16 & 15 & 10 & 10 \\
\hline K & 6 & 5 & 8 & 6 \\
\hline L & 14 & 7 & 9 & 8 \\
\hline M & 14 & 5 & 9 & 5 \\
\hline N & 10 & 3 & 7 & 7 \\
\hline O & 25 & 10 & 8 & 8 \\
\hline
\end{tabular}

Fonte: Autores (2021)

Na Figura 3 pode ser observado que o leiaute da produção não permitia uma circulação de mercadoria de forma otimizada e o fluxo de movimentação, ocasionava gargalos de posição entre os operadores. A movimentação dos equipamentos entre os operadores, que anteriormente feita por caixas plásticas foi substituída por esteira mecânica dotada de rodízios, eliminando o tempo de espera para movimentação e o estoque entre operadores. 
Com a adequação do novo leiaute, apresentado na Figura 4, nota-se uma melhora significativa no fluxo de materiais e redução de operadores, além de possibilitar um fluxo contínuo e lógico, otimizando movimentações e montagem dos equipamentos.

Figura 3 - Layout da Célula de trabalho e produção (antes)

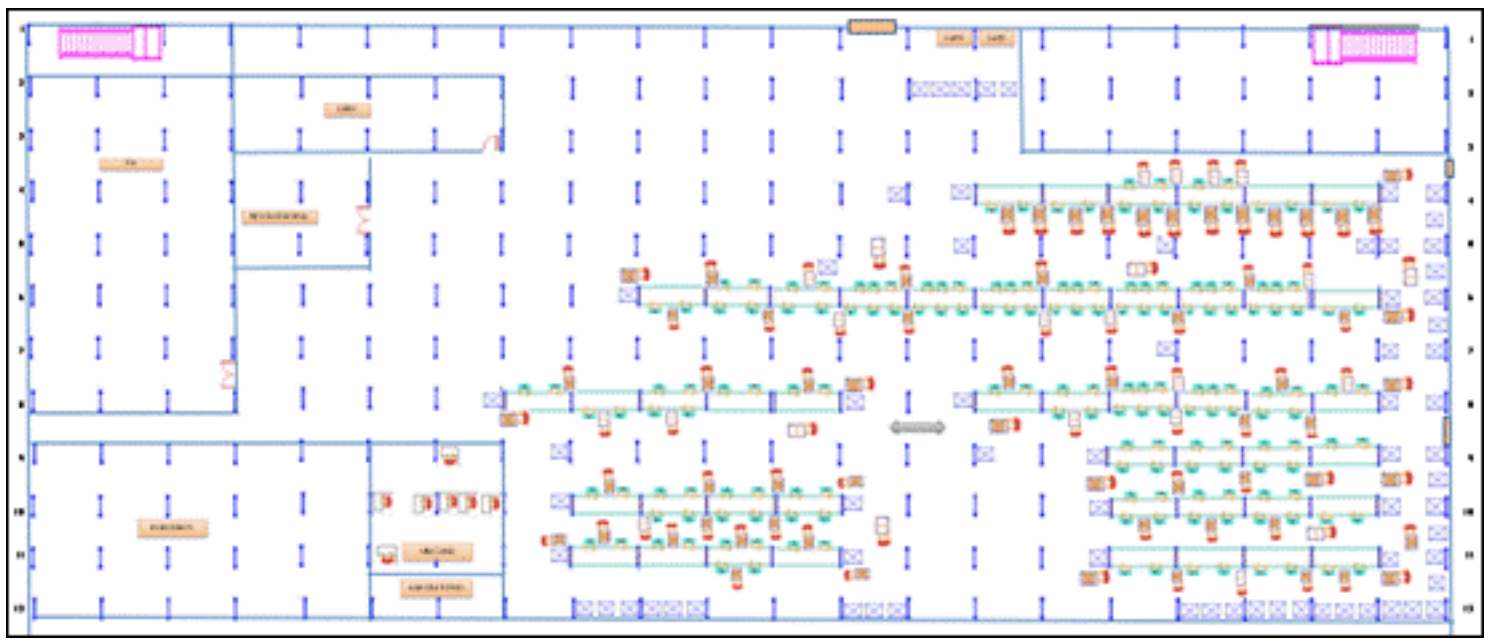

Fonte: Autores (2021)

Figura 4 - Layout da Célula de trabalho e produção (após a melhoria)

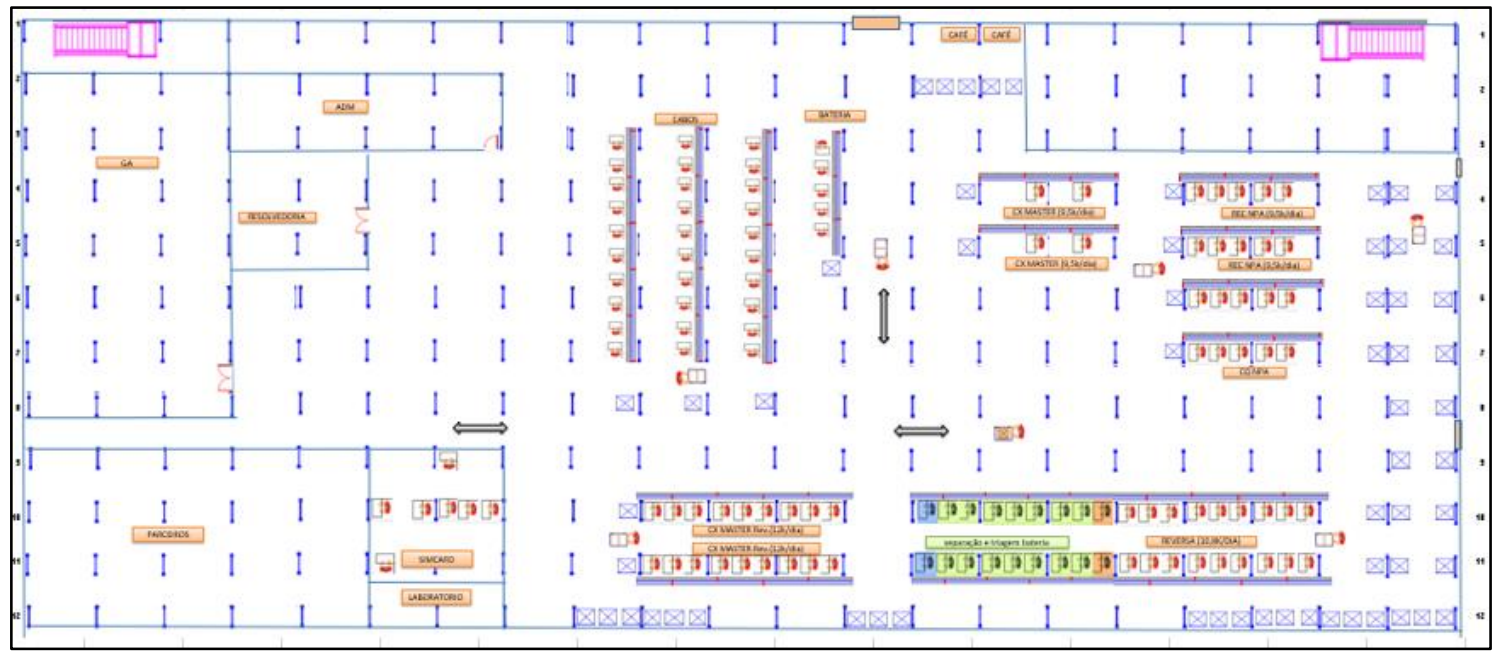

Fonte: Autores (2021)

Associado a implantação das melhorias citadas anteriormente, foi elaborado o mapeamento de processo apresentado na Figura 5, com o mapeamento, foi possível identificar as atividades que não agregam valor ao processo, fornecendo dados para elaboração de um novo projeto de linha de montagem, onde algumas foram eliminadas outras incorporadas, resultando em redução de dez atividades, proporcionando um ganho 
significativo para o processo.

Figura 5 - Mapeamento do Fluxo de Valor (Processo)

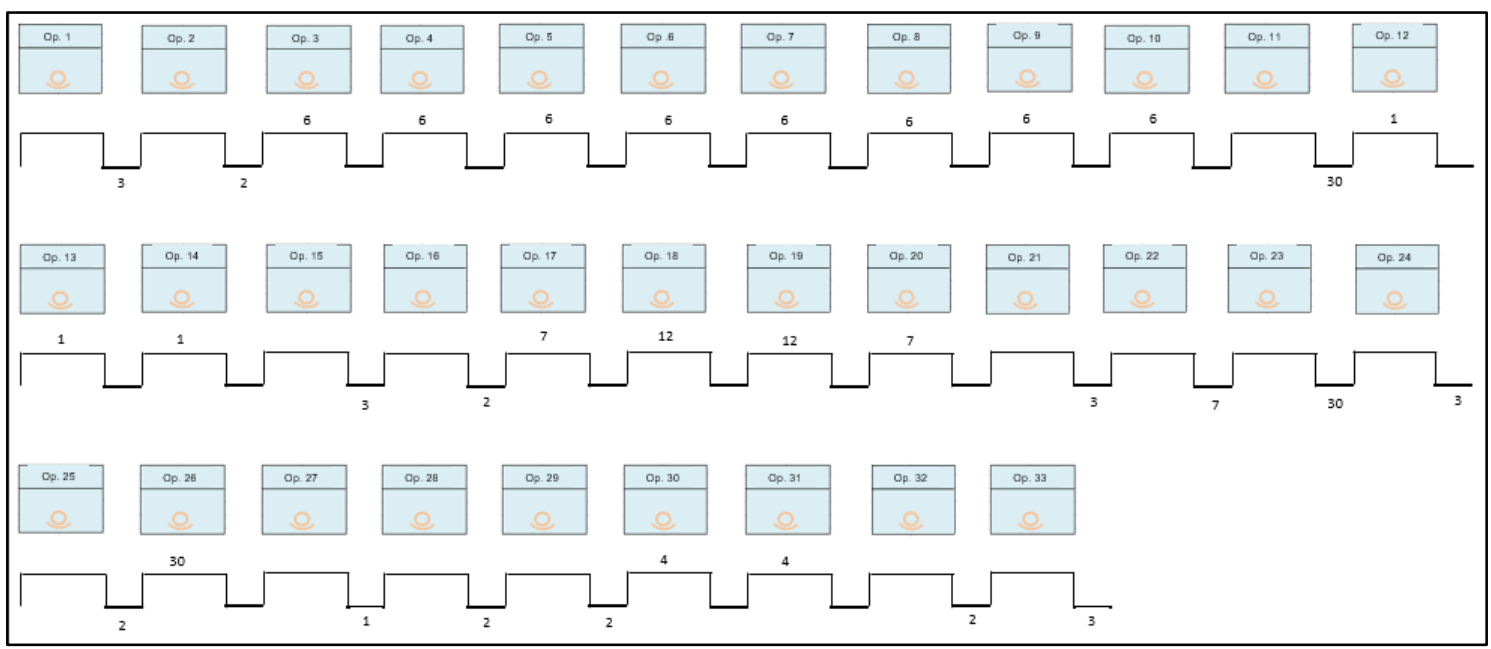

Fonte: Autores (2021)

Com a implementação do novo projeto da linha, um novo mapeamento foi realizado e calculado um novo índice de valor agregado, melhorando de 57\% para 85\%, como apresentado na Figura 6.

Figura 6 - Índice de valor agregado do processo

\begin{tabular}{|c|c|c|c|c|}
\hline \multicolumn{3}{|c|}{ MAPEAMENTO DE PROCESSO } \\
\hline & \multicolumn{2}{|c|}{ Antes } & \multicolumn{2}{c|}{ Depois } \\
\hline & VA & VNA & VA & VNA \\
\hline & 127 & 95 & 97 & 17 \\
\hline Total (seg) & \multicolumn{2}{|c|}{222} & \multicolumn{2}{|c|}{114} \\
\hline \hline Índice de VA & \multicolumn{2}{|c|}{$57 \%$} & \multicolumn{2}{|c|}{$85 \%$} \\
\hline
\end{tabular}

Fonte: Autores (2021)

\section{CONSIDERAÇÕES FINAIS}

Com a adoção da metodologia DFMA foi possível analisar o processo atual, identificar oportunidades de melhorias, elaborar cenários para aprovação da alta direção evidenciando os ganhos preliminarmente projetados.

A proposta do DFMA é de proporcionar redução de desperdícios nos processos, redução de ferramentas e de processos, além da simplificação dos processos. Neste estudo 
com a aplicação das ferramentas de VSM, análise de tempos e movimentos e estudo de leiaute de maneira simultânea, proporcionou alguns ganhos: uma redução geral de $33 \%$ dos operadores, com um melhor balanceamento da linha; uma redução total de $14 \%$ das operações, o que demonstrou a possibilidade de eliminação do turno adicional; as modificações no leiaute reduziu o número de movimentações e do tempo do ciclo, e otimizar o tempo de atravessamento; e por fim o índice de valor agregado que subiu de 57 para $85 \%$.

Os resultados alcançados e citados acima, foram construídos pelos conceitos de DFMA, em facilitar o manuseio, simplificação dos movimentos e operações, e que afetam diretamente na redução dos custos no desenvolvimento e na montagem, proporcionando redução substancial no custo do produto.

As melhorias implementadas foram acompanhadas e validadas pela alta direção juntamente com o cliente, o qual, ao constatar o real aumento da capacidade produtiva, redução do prazo de entrega e flexibilidade para absorver alteração de demanda, vem aumentando gradativamente a colocação de pedidos fortalecendo a relação comercial entre as empresas.

\section{REFERÊNCIAS}

BOKRANTZ, J., SKOOGH, A., YLIPAA, T.; STAHRE, J. Handling of production disturbances in the manufacturing industry. Journal of Manufacturing Technology Management, v. 27, n. 8, p.1054-1075, 1 jan. 2016.

BOOTHROYD, G. Product design for manufacture and assembly, Computer-Aided Design, v. 26, n. 8, p. 505-520.

CARMO, H. M. O. DO. GESTÃO DE RECEBÍVEIS: UMA ANÁLISE DA OFERTA DE MAQUINETAS DE CARTÃO PARA MICROEMPREENDEDORES INDIVIDUAIS. Revista Livre de Sustentabilidade e Empreendedorismo, v. 2, n. 2, p. 28-41, 2017.

CORREA, C. A.; CORRÊA, H. L. Administração de Produção e Operações. São Paulo: ATLAS, 2017.

DRISCOLL, M.O. Journal of Materials Processing Technology, v. 122, p. 318-321, 2002.

FERGUSON, S. M.; OLEWNIK, A.T.; CORMIER, P. A review of mass customization across marketing, engineering and distribution domains toward development of a process framework, Research in Engineering Design, v. 25, n. 1, p.11-30, 2013. 
GAITHER, N.; FRAZIER, G. Administración de producción y operaciones 2000.

JASTI, N. V. K.; SHARMA, A. International Journal of Lean Six Sigma, 2014.

JIN, H. W.; DOOLEN, T. L., A comparison of Korean and US continuous improvement projects. International Journal of Productivity and Performance Management, v. 63, n. 4, p. 384-405, 2014.

KIM, G.J.; BEKEY, G.A.; GOLDBERG, K. Y. A Shape Metric for Design-forAssembly IEEE International Conference on Robotics and Automation, pp. 5, 1992.

KOUFTEROS, X.; Lu, G., Peters, R. C., Lai, K.-h., Wong, C. W. and Cheng, T. E., Product development practices, manufacturing practices, and performance: A mediational perspective, International Journal of Production Economics, v. 156, p. 83-97, 2014.

KRUMENAUER, F.Z.; MATAYOSHI, C. T.; da SILVA, I. B.; FILHO, M. S.; BATALHA, G. F., Journal of Achievements in Materials and Manufacturing Engineering, v.31, p. 690-698, 2008.

LEÃO, L. B.; SOTTO, E. C. S. A EVOLUÇÃO DOS MEIOS DE PAGAMENTO.

Revista Interface Tecnológica, v. 16, n. 1, p. 221-232, 30 jun. 2019.

LIBRELATO et al., 2014; RAHANI; AL-ASHRAF, 2012). - Desenvolvimento de Jogo para Simulação de Aprendizagem do Mapa Fluxo de Valor com Materiais Alternativos.

MARTINS, P.; LAUGENI, F., Administração da produção. $3^{\text {a }}$ ed. São Paulo: Saraiva, 2015.

MATTOS, M. A. B. B. A verticalização e exclusividade dos arranjos de meios de pagamento brasileiros. Monografia (Instituto de Economia) - Universidade Federal do Rio de Janeiro, 2017.

PEINADO, J.; GRAEML, A. R. - Operações industriais e de Serviços 2007.

SELVARAJ, P.; RADHAKRISHNAN, P.; ADITHAN, M. An integrated approach to design for manufacturing and assembly based on reduction of product development time and cost. International Journal of Advanced Manufacturing Technology, v.42, p. 13-29, 2008.

SLACK, N.; CHAMBERS, S.; JOHNSTON, R.; BETTS, A. Gerenciamento de Operações e de Processos. Princípios e práticas de impacto estratégico. $2^{\mathrm{a}}$ ed. São Paulo: Bookman, 2013. 
TODIC, V.; VUKMAN, J., Journal of Production Engineer, v.16, p. 47-5, 2012.

Recebido em: 15/10/2021

Aprovado em: 15/11/2021

Publicado em: 20/11/2021 